\title{
Speech
}

\section{Diversity v. globalization: traditional foods at the epicentre*}

\author{
First published online 20 February 2012
}

Ladies and Gentlemen, dear Colleagues,

Today is one of the most important moments in my professional life and I am most grateful to all of you for sharing this with me.

Globalization in food production and consumption is well recognized. I remember, some 10 years ago, when the respected newspaper Economist wanted to establish an index for PPP (the purchasing power parity); they used the price of an international commodity, the Big Mac of the MacDonald's company, as some sort of a standard. There is no denial that globalization is an ongoing process, unavoidable and, in some aspects, even desirable. It entails, however, some risks and disadvantages which can best be demonstrated by looking at the value and the fate of sustainable diets and traditional foods across the globe.

But let us first review some terms. I will briefly refer to the terms diversity, globalization and traditional foods and the cross-cutting issue of sustainable diets. Next, I will consider the health effects of traditional foods and their role in protecting biodiversity.

According to the Food and Agriculture Organization (FAO), sustainable diets are those with low environmental impact which contribute to food and nutrition security and to healthy life for present and future generations. Ideally, sustainable diets are protective and respectful of biodiversity; culturally acceptable, accessible and economically affordable; and nutritionally adequate, safe and healthy.

Globalization refers to the increasing integration of the world's economy through the reduction of various barriers in international trade. Through globalization, regional economies, societies and cultures have become integrated by communication, transportation and trade. The term also applies to the international circulation of ideas and cultural aspects. Globalization is driven by a combination of economic, technological, socio-cultural and political factors.

When I checked for a definition of biodiversity, I liked the following one: 'the degree of variation of life forms within a given ecosystem, biome, or the entire planet'.

Let us now examine what we mean by traditional foods. According to the European Union EuroFIR project,

* On 29 October 2011, Professor Antonia Trichopoulou received the Federation of the European Nutrition Societies (FENS) Award, the Federation's highest distinction, presented every four years to a scientist with research contributions of international acclaim in the field of nutrition. At the presentation of the Award, during the closing ceremony of the 11th International Conference of the Federation, held in Madrid, Spain, Professor Trichopoulou addressed the participants with this lecture on the topic of diversity $v$. globalization in food production and consumption. 'traditional foods' refer to practices or specifications established prior to the Second World War (Fig. 1). The selected time limit 'prior to the Second World War' implies 'prior to the era of mass food production' and it delineates the period when most population groups still applied simple, time-honoured approaches. This is before the large-scale introduction of technological innovations that substantially altered food production processes.

Patterns of food production and consumption have changed in ways that profoundly affect ecosystems and human diets. In fact, the accelerated speed of loss of food biodiversity and degradation of most ecosystems forces us to rethink agricultural systems and diets.

In the FAO's Millennium Development Goals, Goal 7 states 'Ensure environmental sustainability'. This could be achieved by incorporating principles of sustainable development into national policies and programmes.

According to several reports, the livestock industry contributes substantially and disproportionally to air and water pollution, land degradation and loss of biodiversity. Indeed, animals fed on grain need more water than grain crops. The result is that producing animalbased food is typically much less efficient than the harvesting of grains, vegetables, legumes, seeds and fruits for direct human consumption. This is an issue we will need to return to, since traditional foods are largely of plant origin.

It is estimated that, today, $15 \%$ of the world's population suffers from hunger and twice as many suffer from micronutrient deficiencies. Simplification of diets, typical of the globalization pattern, leads to diets low in variety but high in energy. These diets do not solve the problems of undernutrition and micronutrient deficiencies but also promote obesity and chronic diseases, particularly among poor segments of the populations in both developed and developing countries.

In the 26th FAO regional conference it was stressed that the promotion of traditional regional foods is a step towards sustainable rural development. It has also been pointed out that the richness of food varieties contributes to biodiversity (Fig. 2).

As most traditional foods are plant-based and integrated to the local biosystem and economy, they are almost by definition environmentally friendly, or at least minimally disturbing. Nutrition globalization will continue, but it is important to preserve diversity and collective identity. Traditional foods can be at the epicentre of this effort. 
Let me now take up the issue of the Mediterranean diet, a diet that encompasses a plethora of traditional foods. If one examines the Mediterranean diet as a model of a sustainable diet, then the issue of biodiversity is almost inherent. In characterizing sustainable diets, including the Mediterranean diet, biodiversity covers the dynamics of

Traditional means conforming to established practice or specifications prior to the Second World War.

Traditional food is a food of a specific feature or features, which distinguish it clearly from other similar products of the same category, in terms of the use of "traditional ingredients" (raw materials or primary products) or "traditional composition" or "traditional type of production and/or processing method".

Trichopoulou et al. 2006 Trends in Food Science \& Technology,

Fig. 1 'Traditional food' definition - EuroFIR

\begin{tabular}{|l|l|}
\hline & \multicolumn{1}{|c|}{ Resource } \\
\hline $\begin{array}{l}\text { Wheat, } \\
\text { cultivated and wild }\end{array}$ & 106 varieties \\
\hline Apricots & More than 140 varieties \\
\hline Grapes & Hundreds of varieties \\
\hline
\end{tabular}

Source: FAO

Fig. 2 Food biodiversity plant and animal species within that ecosystem, and the genetic resources within species.

In 2010, in a FAO report on biodiversity in sustainable diets, it was stated that: 'Countries, communities and cultures that maintain their own traditional food systems are better able to conserve local food specialties with a corresponding diversity of crop varieties and animal breeds. They are also more likely to show a lower prevalence of diet-related chronic diseases. The Mediterranean diet offers a clear example'.

The importance of the Mediterranean diet for the rest of the world lies less in its specific foods and nutrients, and more in the methods used to capture its essence and the philosophy of sustainability which lies at its core. These same methods can be used to characterize sustainable diets in other ecosystems and food systems. A useful example comes from the 5th AFROFOODS Meeting held in Dakar in December 2009, where the delegates noted that the degradation of ecosystems and the loss of food biodiversity are contributing greatly to the increases in poverty and malnutrition in Africa. They recognized that returning to local crops and traditional food systems is essential for the conservation of biodiversity for food and nutrition, and they stressed that local foods are the basis for African sustainable diets. The way the Mediterranean diet has been conceptualized and studied in relation to health and other parameters can be useful as one of the models for the study of sustainable diets and a reference for addressing some of the challenges faced in many of the developing regions around the globe.

In the 9th century $\mathrm{AD}$, referring to religious faith, the philosopher Scotus wrote: 'Quae vere praedicantur credere, et quae vere creduntur intelligere', which can be approximately translated as 'We should believe the truth as we have experienced it, then try to understand

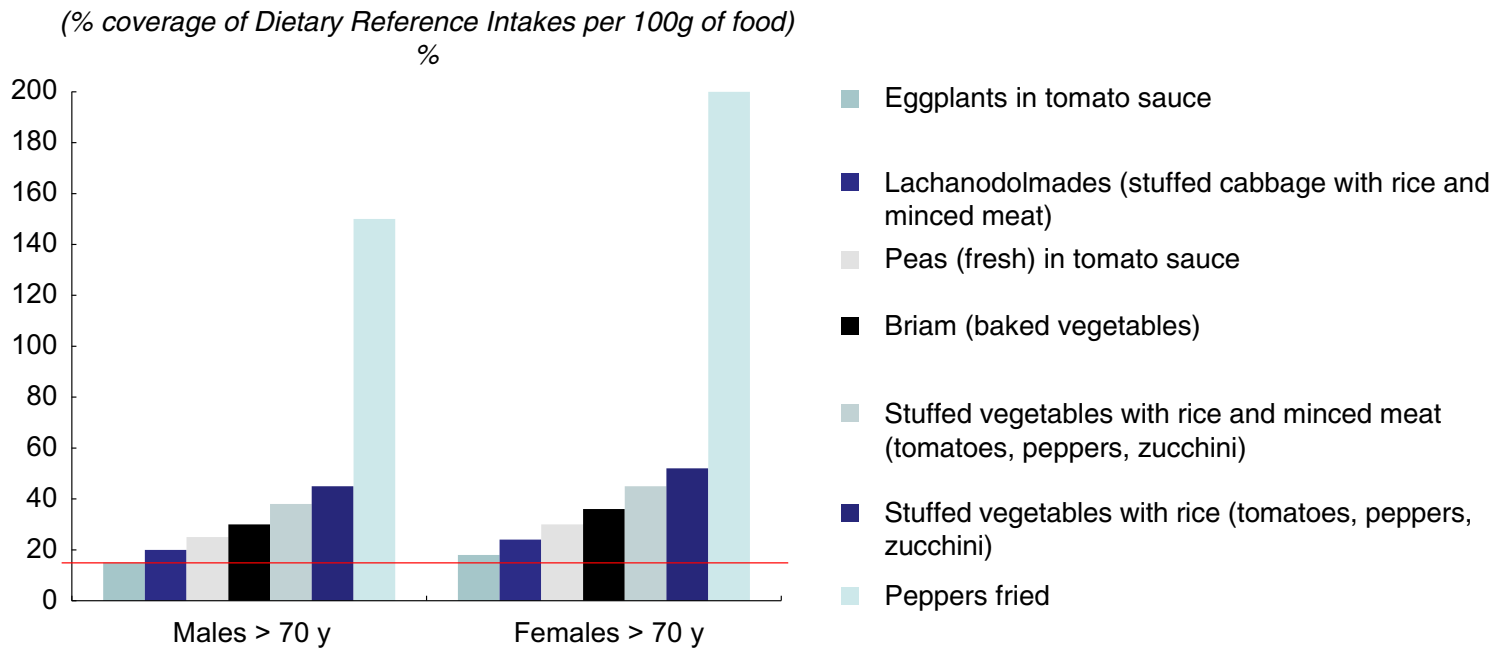

E. Vasilopoulou and A. Trichopoulou. Mediterr J Nutr Metab.2009

Fig. 3 Identification of Greek traditional foods potentially rich in vitamin C for the elderly population group 


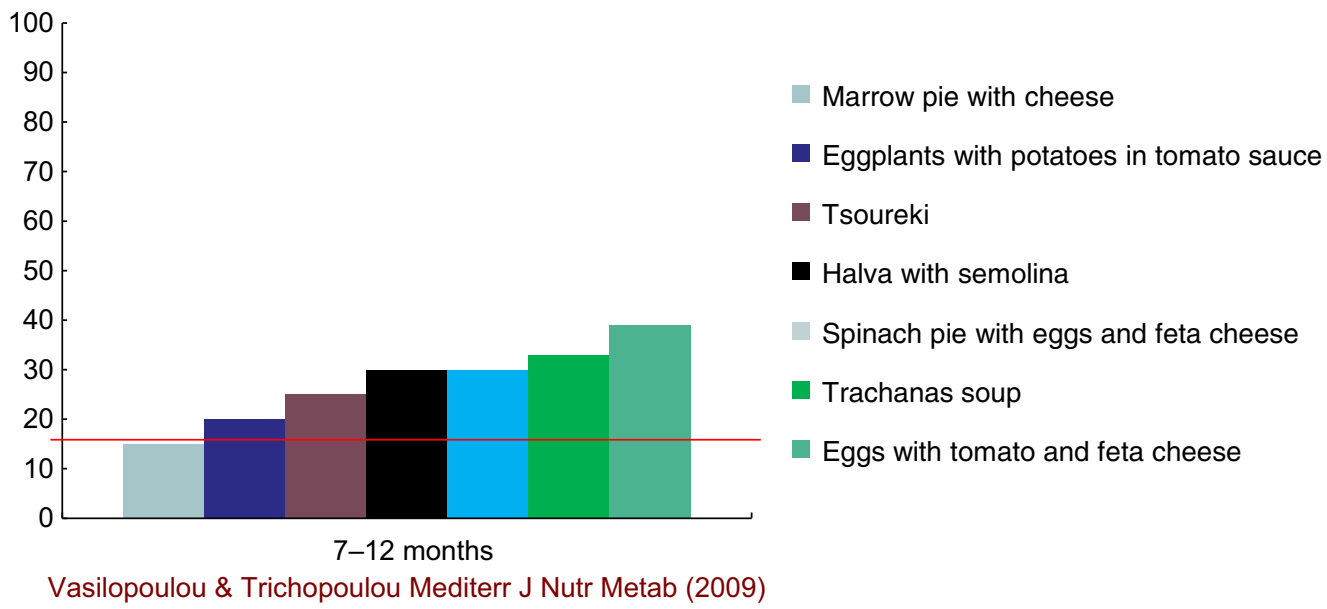

Fig. 4 Identification of Greek traditional foods potentially rich in vitamin E for the infant population group

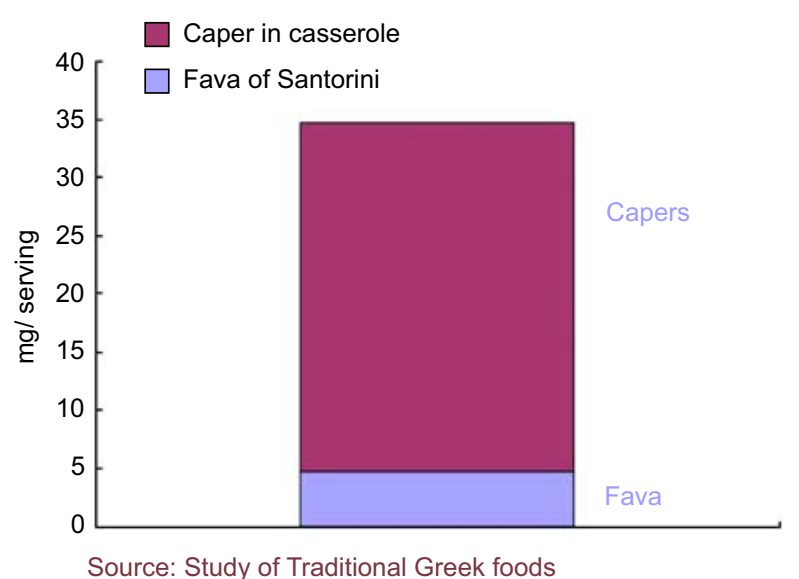

Fig. 5 The flavonol, flavone and flavan-3-ol content of a typical traditional serving of fava complemented with 'caper in casserole' sauce

and explain what we have believed'. Indeed, in the Mediterranean region, there has always been a strong, albeit undocumented, belief that Mediterranean lifestyle is an elixir of well-being. Although one cannot take case reports or the so-called popular wisdom at face value, it is nevertheless intriguing that most centenarians in the Mediterranean region have been inclined to attribute their longevity to their diet and their lifestyle in general.

The health effects of the Mediterranean diet, and indeed its identity, can be partly attributed to the traditional foods which this diet integrates. For the production of traditional foods, local products are generally used. Cultivation of local products contributes to a sustainable environment and employment of local people. The Mediterranean diet, a system rooted in respect for the territory, ensures the conservation and development of traditional activities and crafts linked to fishing and farming, thereby guaranteeing the balance between the territory and the people.
In 2010 the Mediterranean diet was inscribed on UNESCO's Lists of Intangible Cultural Heritage. The term 'cultural heritage' does not end at monuments and collections of masterful objects. It also includes traditions or living expressions inherited from our ancestors and passed on to our descendants, such as oral traditions, performing arts, social practices, rituals and festive events.

Nutritional investigations have provided strong indications that a diet that adheres to the principles of the traditional Mediterranean one is associated with longer survival. Again, this could be partly attributed to Mediterranean traditional foods, which are critical components of this diet.

Our research team has been working with the objective to formulate a multifaceted framework for the systematic investigation of traditional foods and recipes, an approach that could be useful in the study of other traditional foods, in other cultural settings.

In the context of the European Union EURRECA project, we have estimated the micronutrient content of traditional Greek foods in relation to professional recommendations. Many of these foods showed a rich micronutrient profile and we have developed an archive listing a total of over a hundred traditional Greek foods and dishes.

Here I present indicative traditional foods rich in vitamins $\mathrm{C}$ and $\mathrm{E}$ (more than 15\% of the RDA per $100 \mathrm{~g}$ of food; Figs 3 and 4).

There are secrets in the traditional foods which are not immediately evident in food composition tables. Here it can be seen how a humble traditional dish, the Santorini island fava, is enriched with flavonol, flavone and flavan-3-ol with 'caper in casserole' sauce (Fig. 5).

We have analysed a weekly menu typical of the dietary pattern of the Greek variant of the Mediterranean diet, largely composed of traditional foods, to examine whether it meets the nutritional recommendations developed by the Scientific Committee for Food of the European Commission. It is clear that the Mediterranean 


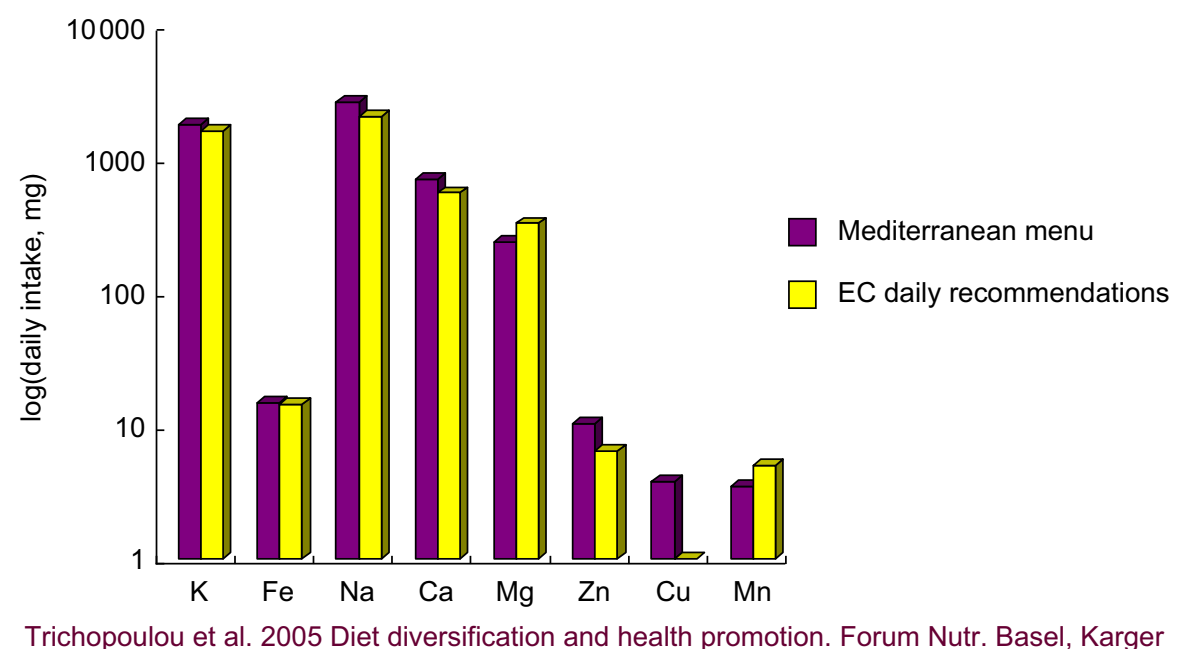

Fig. 6 Inorganic constituents of a weekly menu typical of the dietary pattern of the Greek variant of the Mediterranean diet and comparison with European recommendations

menu is fully compatible with these recommendations (Fig. 6).

Are there other traditional diets with similar environmental and perhaps health benefits? Most likely yes; and they should be treated as sustainable diets by the respective populations. I have talked specifically about the Mediterranean diet because of familiarity with it and also because, in my opinion, it is a dietary pattern that can be adopted easily by modern Western societies.

More generally, however, there is a need to highlight biodiversity, food production and food consumption as interconnected elements, with the purpose of promoting a broader assessment of the link between local food products, nutrition, food safety and sustainability with traditional foods at the epicentre.

My dear colleagues thank you for honouring me with your attendance.

Prof. Antonia Trichopoulou, MD, PhD Director, WHO Collaborating Center for Food and Nutrition Policies, Greece Vice President, Hellenic Health Foundation Email: atrichopoulou@hhf-greece.gr

\section{Bibliography}

1. Food and Agriculture Organization of the United Nations (2006) Livestock a major threat to environment. Remedies urgently needed. FAONewsroom, 29 November. http:// www.fao.org/newsroom/en/news/2006/1000448/index.html (accessed September 2011).

2. Food and Agriculture Organization of the United Nations (2011) FAO and the Eight Millennium Development Goals. http://www.fao.org/mdg/en/ (accessed September 2011).

3. Food and Agriculture Organization of the United Nations (2011) International Scientific Symposium on Biodiversity and Sustainable Diets. United Against Hunger. 3-5 November 2010. http://www.fao.org/ag/humannutrition/biodiversity/ meetings/en/ (accessed September 2011).

4. Trichopoulou A, Vasilopoulou E, Georga K et al. (2006) Traditional foods: why and how to sustain them. Trends Food Sci Technol 17, 498-504.

5. Vasilopoulou E \& Trichopoulou A (2009) The micronutrient content of traditional Greek foods. Mediterr J Nutr Metab 2, 97-102.

6. Trichopoulou A, Vasilopoulou E \& Georga K (2005) Diet diversification and health promotion. In Forum Nutr, vol. 57, pp. 135-146 [I Elmadfa, editor]. Basel: Karger. 